\title{
Investigation of the Effect of a dc Glow Discharge on the Performance of a Particle Beam Liquid Chromatography/ Mass Spectrometry Interface
}

\author{
Thomas A. Bellar, William L. Budde, and David D. Kryak \\ U.S. Environumental Protection Agency, Office of Research and Development, Environmental Monitoring \\ Systems Laboratory, Cincinnati, Ohio, USA
}

\begin{abstract}
A low voltage (180-V) dc glow discharge device was inserted just below the pneumatic nebulizer in a particle beam interface of a high performance liquid chromatography/mass spectrometry system. The discharge in a helium atmosphere increased the signal produced by 12 test compounds by factors of about $2-6$. The increases in signal were probably produced by an increase in the efficiency of solute transmission through the interface. The signal increases caused by the glow discharge were compared to somewhat larger increases caused by $0.01-\mathrm{M}$ ammonium acetate in the mobile phase. The combination of glow discharge and ammonium acetate provided no meaningful advantage over the individual techniques. The mechanism of improved transport efficiency is not proven, but the neutralization of charged particles is a viable hypothesis. (J Am Soc Mass Spectrom 1994, 5, 908-912)
\end{abstract}

$\mathrm{W}$ ilkes et al. [1] reported that placement of an alternating current corona discharge near the orifice of the thermospray nebulizer in a liquid chromatography/particle beam/mass spectrometry $(\mathrm{LC} / \mathrm{PB} / \mathrm{MS})$ interface dramatically increased the efficiency of transmission of the compound benzidine through the interface. They attributed this increase to an aerosol neutralizing action of the corona discharge. Their hypothesis was that neutralization of charged particles from the thermospray nebulizer increased the efficiency of particle transport through the interface into the conventional electron ionization (EI) ion source.

Intrigued by the possibility of increasing the throughput of nonvolatile and thermally labile compounds and thereby giving a needed boost to the sensitivity of LC/PB/MS, we conducted experiments with a somewhat different PB interface design. In contrast to Wilkes and co-workers, who used a thermospray nebulizer and an ac discharge, we used a helium-driven pneumatic nebulizer and a low voltage $(180-\mathrm{V}) \mathrm{dc}$ glow discharge. This system was tested with 12 compounds separated in a gradient elution with high performance liquid chromatography. Enhancements observed with the dc glow discharge were compared with slightly greater enhancements [2] caused by ammonium acetate. No effect was observed with an ac discharge. The neutralizing action hypothesis of a glow discharge is neither proved nor dis-

Address reprint requests to Dr. William L. Budde, U. S. E. P. A., 26 W. Martin L. King Drive, Cincinnati, OH 45268. proved, but the ammonium acetate enhancement remains consistent with the mass transport hypothesis [3].

\section{Experimental}

\section{Materials}

Compounds used in this work were the highest purity materials available from commercial sources ${ }^{1}$ or from the U.S. Environmental Protection Agency's Repository for Toxic and Hazardous Materials, and were used without purification. Acetonitrile was Burdick and Jackson (Muskegon, MI) UV grade, and reagent water was obtained from a Millipore (Bedford, MA) Milli-Q water purification system. Ammonium acetate was American Chemical Society reagent grade. High purity helium was obtained from either the U.S. Bureau of Mines or Linde, Inc. (Somerset, NJ).

\section{Instrumentation}

This work was conducted with an instrument system that consisted of a Hewlett-Packard (HP; Avondale, PA) model 1090 high-performance liquid chromatograph equipped with an autoinjector, a $\mathrm{HP}$ model $59980 \mathrm{~A}$ particle beam interface, a HP model 5988A quadrupole mass spectrometer, and a HP RTE-A data

\footnotetext{
${ }^{1}$ Mention of trade names or commercial products does not constitute endorsement or recommendation for use.
} 
system. Post-column additions to the mobile phase were accomplished with a HP model 1090 pump and a Valco (Houston, TX) $\mathrm{T}$ connection. The particle beam interface consisted of a concentric pneumatic nebulizer, a metal-walled heated desolvation chamber, and a two-stage momentum separator.

The interface was modified by insertion of a glass liner (8.0-mm o.d.; $5.65-\mathrm{mm}$ i.d.) inside the entire length (about $30 \mathrm{~cm}$ ) of the metal direct insertion probe that carried the particle beam from the momentum separator to the ion source. The glass liner was tapered (5.7-mm o.d.; 4-mm i.d.) to fit into the ion source entrance hole and was inserted to provide insulation between charged particles and the metal probe wall. The effectiveness of this liner was not evaluated.

The PB interface was further modified by removal of the drain fittings at the base of the desolvation chamber just below the pneumatic nebulizer and insertion of a low voltage dc glow discharge probe into the hole. The construction details and dimensions of the glow discharge device are shown in Figure 1. The tip of the discharge device was about $2 \mathrm{~cm}$ below the spray and was powered by a BioRad (Mountain View, CA) model $500 / 200$ dc power supply. The stainless steel rod at the center of the discharge device was tapered to a point. The 1.6-mm-i.d. $\times 6.4-\mathrm{mm}-\mathrm{o}$.d. glass-filled Teflon $(9$ ferrule at the base of the $3-\mathrm{mm}$ Pyrex $B$ tube was sealed around the rod and was slightly grooved or notched to accept the 3-mm tube and to prevent arcing at the base. The spectrometer was equipped with a conventional EI ion source and a conventional electron multiplier detector.

\section{Liquid Chromatography}

The LC column was a Waters (Milford, MA) Novapack 15-cm $\times 2-\mathrm{mm}$ (i.d.) column packed with spherical $4-\mu \mathrm{m}$ silica particles with octadecyldimethylsilyl groups chemically bonded to the silica surface (C18 reverse phase column). An Upchurch (Oak Harbor, WA) C18 guard column or a $2-\mu \mathrm{m}$ stainless steel frit were used to protect the analytical column. The col- umn was conditioned by pumping a $75 \%$ acetonitrile-25\% water $(\mathrm{v} / \mathrm{v})$ solution through it for several hours to remove residual impurities, column bleed, and so forth. The mobile phase for all experiments was a mixture of acetonitrile and water. The solvents were degassed in an ultrasonic bath under reduced pressure and continuously purged with helium at $30 \mathrm{~mL} / \mathrm{min}$ to minimize dissolved air. The injection volume was 6 $\mu \mathrm{L}$ and all injections were made with the autoinjector.

The compounds were separated with a gradient elution formed by mixing solutions A [ $80 \%$ water- $20 \%$ acetonitrile $(\mathrm{v} / \mathrm{v})]$ and $\mathrm{B}(100 \%$ acetonitrile). The flow rate of the mobile phase through the column was 0.3 $\mathrm{mL} / \mathrm{min}$ and the composition was initially $100 \% \mathrm{~A}$, then programmed linearly to $47 \% \mathrm{~A}$ and $53 \% \mathrm{~B}$ after $20 \mathrm{~min}_{\mathrm{s}}$ and back to $100 \% \mathrm{~A}$ after $30 \mathrm{~min}$. Post-column additions were made at a rate of $0.1 \mathrm{~mL} / \mathrm{min}$ of either solution C $[80 \%$ acetonitrile-20\% water $(v / v)]$ or 0.01 $\mathrm{M}$ ammonium acetate in solution $\mathrm{C}$.

\section{Particle Beam and Mass Spectrometry Conditions}

The desolvation chamber was maintained at $55^{\circ} \mathrm{C}$ and the nebulizer and momentum separator were warmed somewhat by contact with the desolvation chamber. The He pressure to the nebulizer was set at $28 \mathrm{lb} /$ in. $^{2}$ and the interface was optimized as previously described [4]. Additional helium was admitted through the discharge probe at a rate of $100 \mathrm{~mL} / \mathrm{min}$ and the dc power supply (Figure 1) was set to $180 \mathrm{~V}$ dc at 20 $\mathrm{mA}$. A violet glow was clearly visible and extended several millimeters from the electrodes. The two-stage momentum separator reduced the pressure to about 0.4 torr at the second stage rough pump. The differentially pumped spectrometer diffusion pumps maintained a pressure of $3-4 \times 10^{-5}$ torr in the manifold surrounding the EI ion source. The ion source temperature was $270{ }^{\circ} \mathrm{C}$ and the electron energy was $70 \mathrm{eV}$. The mass spectrometer was repetitively scanned from 62 to $460 \mathrm{u}$ at $1-2 \mathrm{~s} / \mathrm{scan}$, which usually gave at least 10 scans per LC peak. All spectra were acquired in the positive ion mode.

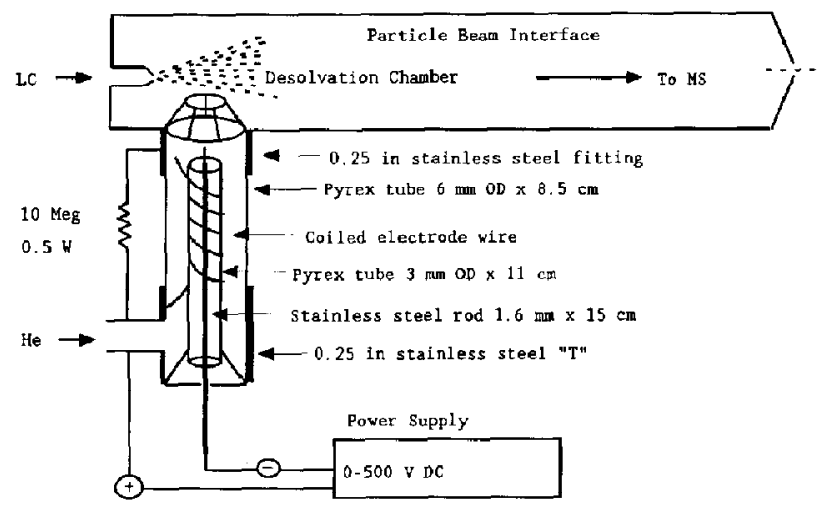

Figure 1. Schematic diagram of the glow discharge device inserted into the particle beam interface. The diagram is not to scale and emphasizes the discharge device. 


\section{Ion Source Tuning and Calibration}

The ion source and lens potentials were adjusted manually to optimize ion intensity from perfluorotributylamine (PFTBA) at mass 219 while a $50 \%$ wateracetonitrile solution passed through the interface. The mass scales were calibrated according to the manufacturers' procedures. Integrations of ion abundances over LC peaks were accomplished automatically by using data system software after specifying a retention time window for each compound and a minimum abundance. Integrated abundances were verified for reasonableness by inspection.

\section{Results and Discussion}

Figure 2 summarizes data from a series of experiments to evaluate the effects of the glow discharge and ammonium acetate independently and together. The compounds listed along the horizontal axis are in elution order, which corresponds to a decreasing percentage of water in the mobile phase. The full names and abbreviations in elution order are given in Table 1 . The bar on the extreme left of each cluster in Figure 2 gives the mean integrated ion abundance of the quantitation ion from three separate measurements of each compound with the discharge off and no post-column addition of ammonium acetate to the mobile phase. The relative standard deviations (RSD) from these measurements (Table 1 under off /off) are all under $20 \%$ and most are less than $10 \%$ except for $3,3^{\prime}$-dichlorobenzidine, which gave a relatively weak signal and a somewhat more variable response because only $6 \mathrm{ng}$ was injected.

The second bar from the left in each cluster in Figure 2 is the mean integrated ion abundance with the discharge on, but again without ammonium acetate present. A violet glow was clearly visible sur-

Figure 2. Mean integrated ion abundances measured for 12 test compounds under various conditions shown in the legend. rounding the rod tip and a significant increase in integrated signal was observed for all compounds with the discharge on. The RSDs for five separate measurements and the mean enhancement factors (EFs) are shown under the heading on/off in Table 1. The EF is the mean integrated ion abundance relative to the mean abundance in the experiment with the discharge off and no ammonium acetate present (off/off). The RSDs are all under $20 \%$ and most are under $10 \%$, but the EFs range from 1.8 to 6.2 with a mean of 4 . The mass spectra of all compounds were identical to those observed with the discharge off. There was no correlation between the EFs and the elution order that corresponds to a decreasing percentage of water in the mobile phase.

The signal enhancements caused by the glow discharge were compared with enhancements caused by adding $0.01-\mathrm{M}$ ammonium acetate to the mobile phase between the end of the chromatography column and the nebulizer [2]. Post-column addition was used to preclude enhanced signals caused merely by improved chromatography in the presence of ammonium acetate and to isolate the effects resulting from improved transport through the PB interface [3]. The ammonium acetate enhancements are shown in the third bar from the left in Figure 2. The RSDs for five separate measurements and the mean EFs are shown under the heading off/on in Table 1. The precision of these measurements is excellent with all RSDs except caffeine below $6.5 \%$. Again, the mass spectra of all compounds were identical to those observed without added ammonium acetate. Post-column addition of ammonium acetate produced a larger mean signal enhancement than the glow discharge for all compounds except linuron. There was no correlation between the EFs and the elution order that corresponds to a decreasing percentage of water in the mobile phase.

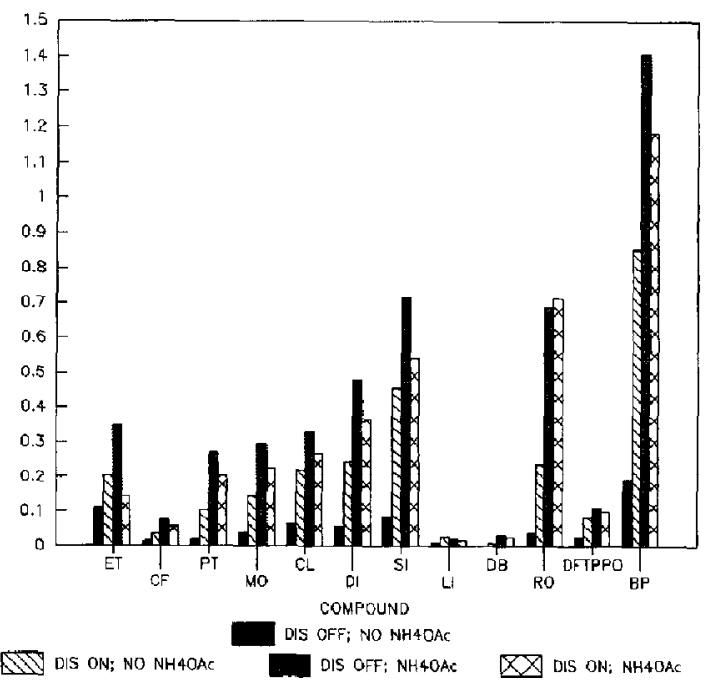


Table 1. Test compounds, abbreviations, amounts, quantitation ions, precision of replicate measurements, and mean enhancement factors

\begin{tabular}{|c|c|c|c|c|c|c|c|c|c|}
\hline \multirow[b]{3}{*}{ Compound } & \multirow{3}{*}{$\begin{array}{l}\text { Amount } \\
\text { injected } \\
\text { (ng) }\end{array}$} & \multirow[b]{3}{*}{$\begin{array}{c}\text { Quant. } \\
\text { ion }\end{array}$} & \multicolumn{7}{|c|}{ Discharge/ammonium acetate } \\
\hline & & & \multirow[b]{2}{*}{$\begin{array}{l}\text { off/off } \\
\text { rsd }^{a}\end{array}$} & \multicolumn{2}{|c|}{ on/off } & \multicolumn{2}{|c|}{ off/on } & \multicolumn{2}{|c|}{ on/on } \\
\hline & & & & $\operatorname{rsd}^{p}$ & $\mathrm{EF}^{\mathrm{C}}$ & $\mathrm{rsd}^{\mathrm{b}}$ & $E F^{E}$ & $\mathrm{rsd}^{d}$ & $E F^{\mathrm{c}}$ \\
\hline \multicolumn{10}{|l|}{ Ethylene thia- } \\
\hline urea $\langle\mathrm{E} T\rangle$ & 384 & 102 & 4.4 & 16 & 1.8 & 4.3 & 3.1 & $\mathbf{e}$ & e \\
\hline Caffeine (CF) & 173 & 194 & 7.1 & 17 & 2.6 & 23 & 5.9 & 0.4 & 4.4 \\
\hline \multicolumn{10}{|l|}{ a-chlorophenyl } \\
\hline thiourea (PT) & 391 & 151 & 6.4 & 7.4 & 5.3 & 1.8 & 14 & 0.1 & 10 \\
\hline Monuron (MO) & 374 & 72 & 4.8 & 11 & 3.6 & 3.5 & 7.4 & 1.6 & 5.7 \\
\hline Carbaryl (CL) & 679 & 144 & 4.8 & 7.0 & 3.7 & 6.5 & 5.6 & 0.1 & 4.5 \\
\hline Diuron (DI) & 300 & 72 & 9.5 & 4.9 & 4.6 & 5.1 & 9.0 & 4.2 & 6.9 \\
\hline Siduron (SI) & 335 & 93 & 7.6 & 6.3 & 5.3 & 2.5 & 8.4 & 1.6 & 6.3 \\
\hline Linuron (LI) & 1140 & 161 & 1.4 & 8.1 & 2.2 & 4.0 & 1.8 & 6.6 & 1.5 \\
\hline \multicolumn{10}{|l|}{ 3,3'-Dichloro } \\
\hline benzidine (DB) & 6 & 252 & 23 & 18 & 5.8 & 3.2 & 26 & 1.6 & 20 \\
\hline Rotenone (RO) & 604 & 192 & 19 & 6.7 & 6.2 & 2.2 & 18 & 1.3 & 19 \\
\hline \multicolumn{10}{|l|}{ phenylphosphine } \\
\hline oxide (DFTPPO) & 600 & 271 & 14 & 3.3 & 3.3 & 1.0 & 4.4 & 1.4 & 3.9 \\
\hline \multicolumn{10}{|l|}{ Benzoylprop } \\
\hline ethy! (BP) & 390 & 105 & 7.8 & 3.4 & 4.4 & 3.3 & 7.3 & 2.2 & 6.1 \\
\hline
\end{tabular}

The effects of the simultaneous presence of the glow discharge and post-column addition of ammonium acetate are shown in the right bars of each cluster in Figure 2. Except for rotenone, there was a small reduction in integrated ion abundances compared to ammonium acetate alone for the test compounds. However, the combined effects were still greater than the enhancements due to the discharge alone except for linuron. The RSDs and EFs for this experiment are shown under the heading on/on in Table 1. Good precision was obtained except for ethylene thiourea, which cannot be reported because of a large undetermined error in one of the measurements. The mass spectra of all compounds were again identical to those measured with the discharge off and no added arnmonium acetate. There was no correlation between the EFs and the elution order that corresponds to a decreasing percentage of water in the mobile phase.

Although diuron and linuron have very similar $\mathrm{N}$-dichlorophenylurea structures that differ only by an oxygen atom between a urea nitrogen and a N-methyl group, and although they elute relatively close in time, their responses to the glow discharge and ammonium acetate are significantly different (Table 1). We speculate that the results for linuron may be anomalous because it gives a relatively weak signal (Figure 2) and a much larger quantity (1140 ng; Table 1) was injected to provide a measurable ion abundance. This larger quantity may have reduced the effectiveness of the discharge and the ammonium acetate.

Wilkes et al. proposed that the corona discharge caused neutralization of charged particles from the thermospray nebulizer and the neutral particles were transported more efficiently through the $\mathrm{PB}$ interface to the conventional EI ion source [1]. Although their instrument system (particularly the thermospray nebulizer, ac discharge, and the membrane gas diffusion cell) was different than the design employed by us, their explanation is plausible and could account for the results reported here.

An alternative and our speculative explanation, however, is that the glow discharge causes charging of some neutral particles that increases the rate of aggregation of particles to form larger particles that are more efficiently transported through the PB interface to the ion source. It has been reported that under somewhat similar conditions (a dc glow discharge at $500-900 \mathrm{~V}$ in $1 \mathrm{~atm}$ of helium), primary and secondary ions are formed and the secondary ions can be measured by direct insertion into a mass spectrometer [5]. These primary and secondary reagent ions could cause charging of some neutral particles and facilitate the aggregation and transport process. This explanation is consistent with the proposed mass transport effect of ammonium acetate in a PB interface [3]. Ammonium acetate and other volatile coeluting substances such as 
isotopically labeled analytes are believed to increase mass transport by forming larger or more dense particles that have more momentum and are more often successful in passing through the PB interface [3].

\section{Conclusion}

The use of a glow discharge to increase throughput in a PB interface may be a viable option that needs additional research. The $P B$ interface has several important advantages over other LC/MS techniques, including the generation of standard EI mass spectra, but improvements in efficiency are needed for many applications. The signal increases provided by the glow discharge are significant and glow discharge may be used to increase efficiency without the complication of adding ammonium acetate. The combination of glow discharge and ammonium acetate provided no meaningful advantage over the individual techniques. The mechanism of improved transport efficiency is not known, but the neutralization of charged particles and/or the formation of larger particles are viable hypotheses. Unfortunately, no further rescarch is planned for the near future to investigate this phenomenon.

\section{References}

1. Wilkes, J. G.; Leonidou, I.; Vestal, M. L. Proceedings of the 40th ASMS Conference on Mass Spectrometry and Allied Topics; Washington, DC, May 31-June 5, 1992, pp. 1294-1295.

2. Bellar, T. A.; Behymer, T. D.; Budde, W. L. J. Am. Soc. Mass Spectrom. 1990, J, 92-98.

3. Ho, J. S.; Behymer, T. D.; Budde, W. I.; Bellar, T. A. J. Am. Soc. Mass Spectrom. 1992, 3, 662-671.

4. Behymer, T. D.; Bellar, T. A.; Budde, W. L. Anal. Chem, 1990, $62,1686-1690$.

5. Zhao, J.; Zhu, J.; Lubman, D. M. Anal. Chem. 1992, 64, $1426-1433$. 